\title{
Genes, Brain, and Language: A brief introduction to the Special Issue
}

Language is a fundamental human ability, a definitive feature of our species. In this Special Issue, we bring together a series of papers that add to our knowledge of how genetic factors may influence language-related networks in the human brain. The papers describe studies using twin and family approaches, neuroimaging, genome wide association scans (GWAS), and candidate-gene analyses, to examine the neurobiology of language from a molecular genetic perspective. The Special Issue is broad in scope, investigating different aspects of genetic contributions to language across animal models and humans, in both healthy and disordered populations.

Although it is obvious that linguistic input is necessary for a language to be learned, twin and family studies have firmly established that language acquisition and proficiency (both for spoken and written language) are subject to substantial genetic influences (Graham et al., 2015). Here, Swagerman et al. report the first general-population study to explore the family resemblance of reading ability using a genetically-sensitive design. By analyzing both twin-sibling and parent-offspring samples, they show that individual differences in reading ability are mainly accounted for by genetic factors. Interestingly, environmental factors shared between parents and children did not contribute to familial resemblance of reading ability, nor was there evidence for cultural transmission from parents to their offspring. Swagerman et al. show that having a familial risk for a reading disability such as developmental dyslexia is unlikely to be attributable to the home literacy environment, and instead strongly reflects the transmission of genetic variants that increase susceptibility. This study, demonstrating the importance of genetic factors for neurobiological pathways underpinning language-related skills, sets the scene nicely for the later papers that zoom in on the particular molecular variants that could be relevant, trying to decipher their effects on brain and behavior.

Neuroimaging measures of brain structure and function provide promising intermediate phenotypes for tracing genetic influences on spoken and written language. Three papers in the Special Issue apply candidate gene and/or GWAS approaches to investigate influences of variants in languagerelated genes on variability in grey matter in relevant regions of the human brain. Importantly, neuroimaging genetics studies depend on large sample sizes (de Zubicaray et al., 2008); the three studies here used cohorts of 1275-1717 participants to help ensure robust findings.

Gialluisi et al. targeted several single-nucleotide polymorphisms (SNPs) that had shown suggestive associations with reading and language skills in prior work. These associations, with the genes CCDC136 (coiled-coil domain containing 136) and FLNC (filamin C) on 7q32.1, and RBFOX2 (RNAbinding protein, fox-1 homolog 2) on 22q12.3, were the strongest findings from the researchers' earlier large-scale GWAS meta-analysis (Gialluisi et al., 2014) in a large sample of individuals with histories of reading or language problems, and their siblings. The authors first performed a literature search to constrain their hypotheses to cortical regions that reliably show differences in individuals with language impairment and/or dyslexia: middle and superior temporal gyri (MTG, STG), postcentral parietal gyrus (PPG), and two sub-regions of the inferior frontal gyrus (IFG; pars opercularis [PO] and triangularis (PT], respectively). In their sample of 1275 healthy participants, the minor allele of the variant rs5995177 within RBFOX2 was associated with a significant reduction in grey matter thickness in left PPG, right MTG and IFG (PO and PT), and bilaterally in the STG. These findings converge with reports of reduced thickness in these regions in dyslexic populations, suggesting the prior findings of association with reading/language performance could potentially be mediated by effects on grey matter thickness in these areas.

In the Special Issue's second neuroimaging genetics study adopting a candidate gene approach, Udden et al. investigated associations between rs7794745, a common SNP in CNTNAP2 (contactin-associated protein-like 2), and variations in regional grey matter volumes. In earlier work, rs7794745 was associated with variation in reading and language abilities, but also with susceptibility to a range of disorders, such as autism. Prior studies of rs7794745 with neuroimaging endophenotypes have used relatively small samples that were likely to be underpowered. Here, Udden et al. sought to replicate the findings from an earlier neuroimaging genetics study of 314 participants that reported reduced grey matter volumes in the bilateral cerebellum, fusiform gyri, occipital and right frontal cortices for carriers of the T allele (Tan et al., 2010), but in this case using a much larger sample (1717 participants). The authors were only able to replicate the association in the left superior occipital gyrus, with carriers of the T allele showing reduced grey matter volume. Udden et al. thus highlight the essential role of independent replication in neuroimaging genetics studies of language-related candidate genes (de Zubicaray et al., 2008).

The third study in the Special Issue to take advantage of both neuroimaging and genetics approaches investigated the etiology of language impairments in older adults at risk for and with a diagnosis of Alzheimer's disease. Deters et al. examined associations between grey matter density and a composite measure of verbal fluency and naming in their sample of 1560 participants from the longitudinal, multi-center Alzheimer's Disease Neuroimaging Initiative study. After first adjusting for episodic memory performance, poorer language performance was significant associated with atrophy in regions of the left temporal, parietal and frontal lobes. A GWAS revealed that variants in GLI3 (GLI family zinc finger 3), a developmental transcription factor involved in patterning brain structures, were significantly associated with language impairments in this sample, the first time that this gene has been connected to language-related functions. One of the associated SNPs (rs3801203) was also associated with lower grey matter 
density predominantly in frontotemporal regions, suggesting the association with language performance could be mediated by this mechanism. It is worth noting that none of the neuroimaging genetics studies in this Special Issue have used formal mediation analyses to model the relationship between gene variants, grey matter findings and language performance in a single sample - this will be an important direction for future investigations in this field.

The final paper in the Special Issue provides an example of how animal models can be used to investigate gene function, targeting candidate genes that have been identified in human studies of language-related traits. Specifically, Rendall et al. investigated the effects of a well-known dyslexia candidate gene on the brain using mouse knockout technology. The researchers assessed the behavioral phenotypes of mice carrying homozygous disruptions of the rodent homolog of DYX1C1 (dyslexia susceptibility 1 candidate 1, now also known as DNAAF1, dynein axonemal assembly factor 4). They used a strategy that enabled the gene to be selectively knocked out in the cortex and hippocampus. Compared to mice in which the gene was intact, those with the DYX1C1 knockout showed impairments in memory and learning, while their performance was relatively unaffected for auditory and motor tasks. Rendall et al. discuss how their mouse model findings may shed further light on the potential contributions of DYX1C1 dysfunction to deficits in learning to read in human individuals with dyslexia.

The studies featured in this Special Issue each contribute in different ways to our understanding of how genetic polymorphisms may influence the neurobiology of language, including investigations focused on oral and written modalities. Yet, it is clear we still have a relatively limited knowledge of the genes that impact on core components of language processing in relation to brain structure and function. Several papers in this Special Issue reflect a burgeoning interest in the use of neuroimaging measures as intermediate phenotypes. While showing promise, such approaches require larger samples than those currently in typical use, in addition to independent replications, and a better understanding of mechanisms of gene action. Further, these strategies will need to be complemented by other research lines, both in animals and humans (Graham et al. 2015), to ultimately bridge the gaps between genes, the development and function of brain circuits, and language.

\section{References}

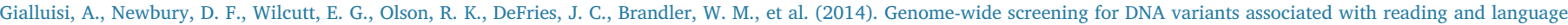
traits. Genes, Brain and Behavior, 13(7), 686-701.

Graham, S. A., Deriziotis, P., \& Fisher, S. E. (2015). Insights into the genetic foundations of human communication. Neuropsychology Review, $25(1)$, 3-26.

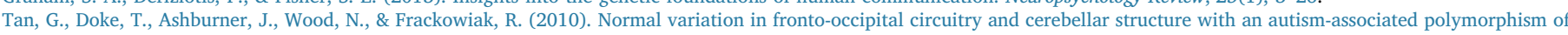
CNTNAP2. NeuroImage, 53(3), 1030-1042.

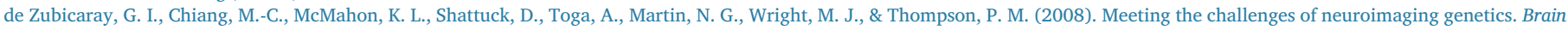
Imaging and Behavior, 2, 258-263.

Greig de Zubicaray

Queensland University of Technology (QUT), Faculty of Health and Institute of Health and Biomedical Innovation, Brisbane, Queensland, 4059, Australia E-mail address: greig.dezubicaray@qut.edu.au

Simon E. Fisher

Language and Genetics Department, Max Planck Institute for Psycholinguistics, Nijmegen, The Netherlands Donders Institute for Brain, Cognition and Behaviour, Radboud University, Nijmegen, The Netherland

E-mail address: simon.fisher@mpi.nl 